\title{
A Consideration of Papillary Squamous Cell Carcinoma of the Gingiva: A Case Study Involving the Immunohistochemistry of Proliferative Activity
}

\author{
Hiroyuki Okada, ${ }^{1}$ Masahiro Hara, ${ }^{2}$ Megumi Yokoyama, ${ }^{1}$ Miya Kato, ${ }^{3}$ Masashi Sakayanagi, ${ }^{3}$ \\ Takashi Kaneda, ${ }^{3}$ Yoshiaki Akimoto, ${ }^{2}$ and Hirotsugu Yamamoto ${ }^{1}$
}

Departments of ${ }^{1}$ Oral Pathology, ${ }^{2}$ Oral Surgery, and ${ }^{3}$ Radiology, Nihon University School of Dentistry at Matsudo, Matsudo, Chiba 271-8587, Japan

Correspondence to :

H. Okada

E-mail : okada.hiroyuki@nihon-u. ac.jp

Keywords :

papillary squamous cell carcinoma, oral, gingiva, immunohistochemistry, proliferative activity, $\mathrm{Ki}^{-67}$

\begin{abstract}
Papillary squamous cell carcinoma (PSCC) is a rare variant of squamous cell carcinoma (SCC) having an exophytic papillary component. We report the case of a 60-year-old woman with oral PSCC of the gingiva. Histopathologically, the operative specimen was composed of exophytic papillary and invasive proliferations of atypical squamous epithelium, from which a pathological diagnosis of PSCC was made. On the $\mathrm{Ki}^{-67}$ labeling index, the exophytic area of PSCC (42.8\%) was higher than the superficial area of SCC (20.7\%) and papilloma (38.9\%). The invasive area of PSCC (53.1\%) was slightly higher than that of SCC (52.2\%). The PSCC had high proliferative activity in both the exophytic and invasive areas, which reflected the PSCC characteristic features of papillary and invasive growths. Clinicians should check for the existence of PSCC in the oromaxillofacial region and provide treatment as they do for SCC.
\end{abstract}

\section{Introduction}

Papillary squamous cell carcinoma (PSCC) is a rare distinct variant of squamous cell carcinoma (SCC) characterized by exophytic and papillary growth with a favorable prognosis (1-4). Papillary squamous cell carcinoma occurs predominantly in men in the 6th and 7th decades and the most common site is the larynx, followed by the oropharynx and nasopharynx $(1,5,6)$. There have been more studies regarding conventional oral SCC (7-9) than oral $\operatorname{PSCC}(6,10)$. Consequently, the biological behavior of oral PSCC is unclear.

We encountered a case of PSCC of the gingiva and studied its proliferative activity to better understand its biological behavior.

\section{Case report}

A 60-year-old woman was referred to us for treatment of a gingival lesion in the lower left molar area. An intraoral examination revealed a reddish, soft papillary mass measuring $28 \times 17 \mathrm{~mm}$ at the edentulous mucosa in the mandibular second premolar and first molar area, and the lesion had slightly spread to the oral floor and buccal mucosa (Fig. 1). The patient did not smoke or drink alcohol. Radiographic examination showed no bone resorption in the area (Fig. 2). Extraoral examination was negative for cervical lymphadenopathy. Computed tomography examination showed no osteolytic change in the area and no cervical lymphadenopathy. Smear cytology revealed atypical squamous epithelial cells suggesting squamous cell carcinoma and the lesion was diagnosed as a Class $\mathrm{V}$ malignant tumor (Fig. 3). An incisional biopsy was taken. Histopathologically, the surface of the specimen was covered by stratified squamous epithelium and cytological and architectural atypia was recognized in up to two-thirds of the epithelium without invasion. Histopathological diagnosis of the biopsy specimen was moderate epithelial dysplasia (Fig. 4). Preoperative laboratory screening was unremarkable except for a slight disorder of hepatic functions 

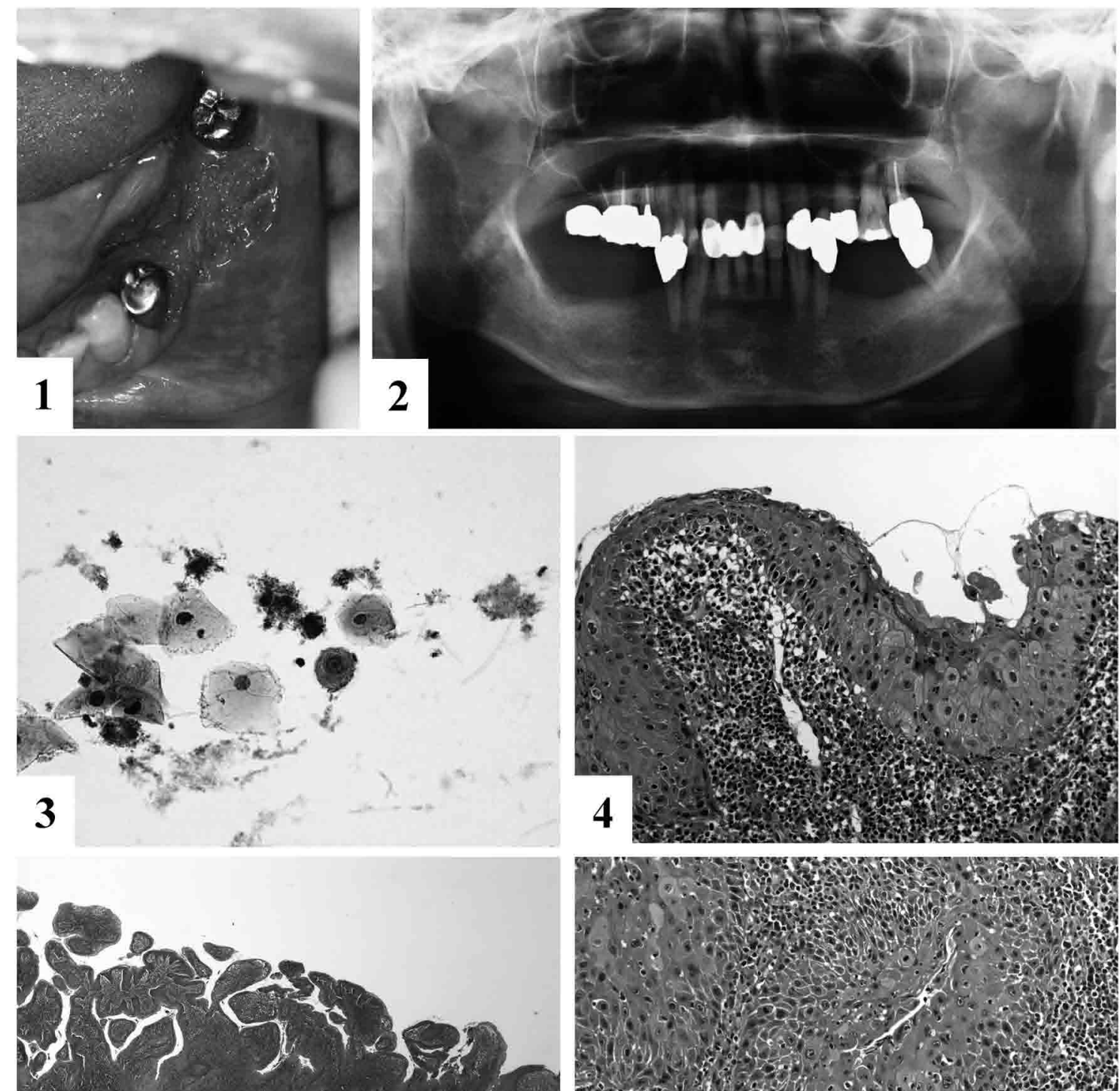

5 (t)
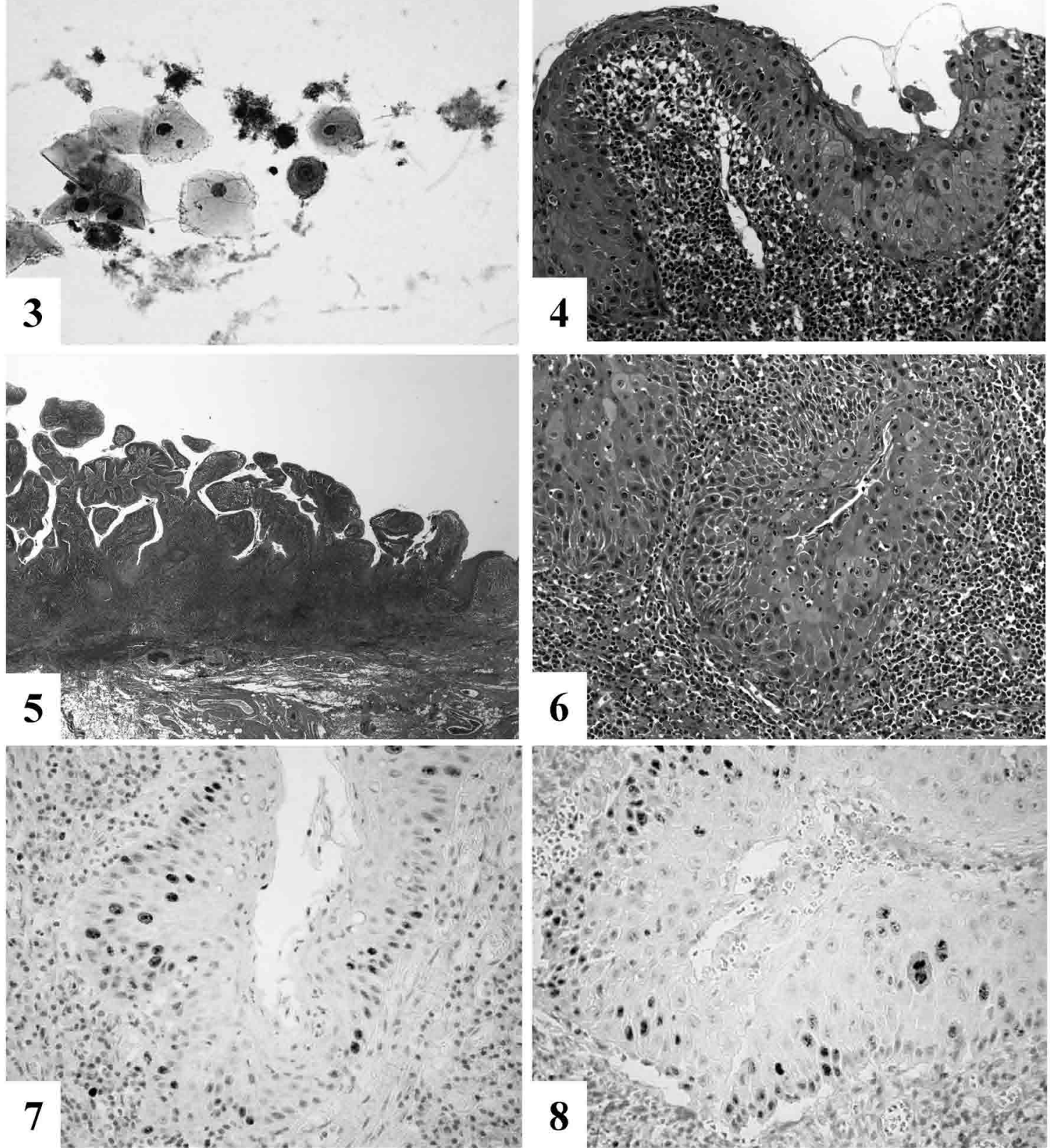
Figure 1. Clinical view showing a reddish, papillary mucosal lesion in the left mandibular second premolar and first molar area (mirror image).

Figure 2. Pantomogram shows no bone resorption in the area.

Figure 3. On smear cytology, atypical keratinized (superficial) and non-keratinized (intermediate and basal) squamous epithelia are recognized (Papanicolaou stain, original magnification $\times 600$ ).

Figure 4. On the biopsy specimen, the surface is covered by stratified squamous epithelium showing atypia in the lower two -thirds of the epithelial thickness (hematoxylin-eosin stain, original magnification $\times 200$ ).

Figure 5. On the surgical specimen, the tumor is composed of exophytic papillary and invasive proliferations of atypical squamous epithelium with stromal lymphocyte infiltration (hematoxylin-eosin stain, original magnification $\times 20$ ).

Figure 6. In the invasive area, atypical squamous epithelium forms islands with anisokaryosis, individual cell keratinization, and mitoses (hematoxylin-eosin stain, original magnification $\times 200$ ).

Figure 7. Many tumor cells positive for $\mathrm{Ki}^{-67}$ in the exophytic area of the papillary squamous cell carcinoma are recognized (original magnification $\times 400$ ).

Figure 8. Many tumor cells in the invasive area of the papillary squamous cell carcinoma are also positivity for Ki-67 (original magnification $\times 400)$.

by hepatitis $\mathrm{C}$ and its treatment. Complete excision of the primary lesion was performed under general anesthesia. Because the intraoperative rapid histopathological diagnosis was SCC, marginal mandibulectomy was further performed, along with extractions of the canine, second premolar, and second molar teeth. As described later, the final histopathological diagnosis was PSCC. The postoperative follow-up was unremarkable and there was no sign of recurrence, metastasis, or cervical lymphadenopathy for 5 years after the operation.

\section{Results}

\section{Histopathological findings}

Macroscopically, the surgical specimen consisted of a grayish-brown mass $35 \times 14 \times 6 \mathrm{~mm}$ in size with a papillary surface.

Histopathologically, the lesion was composed of exophytic papillary proliferation and invasive proliferation that formed islands with pearl formation to the subepithelial connective tissue of the atypical squamous epithelium (Fig. 5). These proliferating tumor cells had one large hyperchromatic nucleus with one or two clear nucleoli (Fig. 6). Anisokaryosis, individual cell keratinization, and mitoses were also recognized (Fig.6). In the stroma, lymphocyte infiltration was prominent.

\section{Proliferative activity}

We divided PSCC and SCC into two areas each: the invasive and the exophytic areas of PSCC and the superficial and the invasive areas of SCC.
The Ki-67 (clone, MIB-1; Dako, Copenhagen, Denmark) labeling index of the exophytic area of PSCC was $42.8 \%$ (Fig. 7) and that of the invasion area of PSCC was 53.1\% (Fig. 8). The Ki-67 labeling index of the superficial area of SCC was $20.7 \%$, that of the invasion area of SCC was $52.2 \%$, and that of the papilloma was $38.9 \%$.

\section{Discussion}

PSCC, a recently described rare subtype of SCC, includes "invasive squamous cell carcinoma that has an exophytic papillary component" $(1,11)$. This tumor has been mostly studied in the field of otorhinolaryngology. The small number of welldocumented cases shows a strong male predilection and the most common site is the larynx, followed by the oropharynx and nasopharynx $(1,5)$. The present PSCC is a very rare case of oral mucosa in a woman. To our knowledge, only three cases of oral PSCC have been reported $(6,11)$.

The diagnostic difficulty of PSCC is well known, because biopsy specimens sometimes display only a partial view of the pathological features. Therefore, a full-thickness biopsy is crucial to allow a definitive histopathologic diagnosis of $\operatorname{PSCC}(1,5,6)$. In the present case, the diagnosis of smear cytology indicated a Class $\mathrm{V}$ malignant tumor, but the diagnosis of SCC was not obtained by incisional biopsy. The biopsy specimen was deemed not to contain the area of invasive SCC. Our experience suggests that smear cytological findings and diagnosis sometimes support the diagnosis from the biopsy because smear 
cytology can collect and observe cells mainly from the surface and middle layers from a large area. Because a correct histopathologic diagnosis is imperative to provide appropriate and effective treatment, we have to judge the gross pathologic change of the lesion and perform a careful and adequate biopsy. Proliferative activity is one of the useful indicators for grading malignancy, possibility of metastasis, and prognosis of the tumor (12). Increased Ki-67 expression has been reported to be a good indicator of cell proliferative activity in premalignant and malignant oral lesions (6). It is reported that PSCC has low (6) or moderate (1) grade malignancy. It is presumed that distant metastasis of PSCC is rare and its prognosis is good $(1,6)$. Takeda et al. (6) reported that PSCC had a high Ki-67 labeling index (53.2$59.0 \%$ ), almost the same as that of SCC (56.7-70.4\%). In the present study, the exophytic area of PSCC (42.8\%) showed a higher $\mathrm{Ki}^{-67}$ labeling index than the superficial area of SCC (20.7\%) and papilloma (38.9\%). Furthermore, the invasive area of PSCC (53.1\%) had a slightly higher Ki-67 labeling index than that of SCC (52.2\%). It is suggested that these high proliferative activities in both the exophytic and invasive areas of PSCC reflect the characteristic features of papillary and invasive growth. Furthermore, the present report clarified that the invasive area of PSCC had high proliferative activity, suggesting that PSCC might have the same grade of malignancy, metastatic rate, and prognosis as SCC in the same stage. In conclusion, clinicians should check for PSCC and provide treatment as they do for SCC.

\section{References}

1. Ferlito A, Devaney KO, Rinaldo A, Putzi MJ. Papillary squamous cell carcinoma versus verrucous squamous cell carcinoma of the head and neck. Ann Otol Rhinol Laryngol, 108: 318-322, 1999.

2. Suarez PA, Adler-Storthz K, Luna MA, El-Naggara K, Aboul-Karim F, Batsakis JG. Papillary squamous cell carcinomas of the upper aerodigestive tract; A clinicopathologic and molecular study. Head Neck,
$22: 360-368,2000$.

3. Mills SE, Gaffey MJ, Frierson HF Jr. Papillary squamous cell carcinoma. In: Rosai J, ed. Atlas of Tumor Pathology. Tumors of the upper aerodigestive tract and ear. Washington DC: Armed Forces Institute of Pathology ; 2000. p.85-88.

4. Johnson N, Franceschi S, Ferlay J, Ramadas K, Schmid S, MacDonald DG, Bouquot JE, Slootweg PJ. Squamous cell carcinoma. In: Barnes L, Everson JW, Reichart P, Sidransky D eds. World Health Organization Classification of Tumours. Pathology and genetics of head and neck tumours. Lyon: IARC Press ; 2005, p.168-175.

5. Ferrer MJ, Estellés E, Villanueva A, López R. Papillary squamous cell carcinoma of the oropharynx. Eur Arch Otorhinolaryngol, 260 : 444-445, 2003.

6. Takeda Y, Satoh M, Nakamura S, Yamamoto H. Papillary squamous cell carcinoma of the oral mucosa; Immunohistochemical comparison with other carcinomas of oral mucosal origin. J Oral Sci, 43: 165-169, 2001.

7. Garzino-Demo P, Dell'Acqua A, Dalmasso P, Fasolis M, La Terra Maggiore GM, Ramieri G, Berrone S, Rampino M, Schena M. Clinicopathological parameters and outcome of 245 patients operated for oral squamous cell carcinoma. J Craniomaxillofac Surg, 34: 344-350, 2006.

8. Pyo SW, Hashimoto M, Kim YS, Kim CH, Lee SH, Johnson KR, Wheelock MJ, Park JU. Expression of E -cadherin, $\mathrm{P}$-cadherin, and $\mathrm{N}$-cadherin in oral squamous cell carcinoma; Correlation with the clinicopathologic features and patient outcome. J Craniomaxillofac Surg, 35 : 1-9, 2006.

9. Hoekstra JW, Kummer JA, van Es RJ. Late $(>5$ years) regional lymph node metastasis of oral squamous cell carcinoma (SCC), proven by p53 mutation analysis. J Craniomaxillofac Surg, 36 : 415-418, 2008.

10. Khan SM, Gossweiler MK, Zunt SL, Edwards MD, Blanchard SB. Papillary squamous cell carcinoma presenting on the gingiva. J Periodontol, $76: 2316^{-}$ 2321, 2005.

11. Ereño C, López JI, Sánchez JM, Bilbao FJ. Papillary squamous cell carcinoma of the larynx. J Laryngol Otol, 115 : 164-166, 2001.

12. Martinez-Barba E, Cortes-Guardiola JA, MinguelaPuras A, Torroba-Caron A, Mendez-Trujillo S, Bermejo-Lopez J. Salivary duct carcinoma ; Clinicopathological and immunohistochemical studies. J Craniomaxillofac Surg, 25 : 328-334, 1997. 